\title{
The Perseverance of Gender Stereotype
}

\author{
Sara Tranquada \\ FCT, M-ITI \\ Portugal \\ sara.tranquada@m-iti.org
}

\author{
Nuno Correia \\ NOVA Lincs, FCT \\ Portugal \\ Nmc@fct.unl.pt
}

\begin{abstract}
Numerous studies have observed unequal representation of gender stereotypes across different areas. However, some of the studies featured in this body of research were focused on using direct measures of implicit associations to understand how students are influenced by gender stereotypes when deciding their work area. This study demonstrates gender stereotypes and their impact, in particular the impact on the number of women in technical fields (e.g. computer science). We present a within-subject experiment, which investigates implicit and explicit gender stereotypes for two areas (exact sciences, and art and humanities). Our results from this study involving 41 students revealed a predisposition to unconsciously attribute men to exact sciences fields (e.g., computer science) and women to arts and humanities, but consciously we observed that in exact sciences, gender attributions were balanced.
\end{abstract}

Gender Bias, Occupational Gender Stereotype, Stereotypes, Implicit Association Test

\section{INTRODUCTION}

"A father and son are in a horrible car crash that kills the dad. The son is rushed to the hospital; just as he's about to go under the knife, the surgeon says, 'I can't operate - that boy is my son!" Who is the surgeon? (Barlow, 2014, pp. 1)

A riddle is often a way to exert the mind, to trick us to reveal our deepest thoughts, all with the purpose of enlightening us with the current events (Literary Devices, 2015). If your answer was the stepparent or even the adoptive parent, then you are part of the astonishing majority that had it wrong. The correct answer is, the mother. Mikaela Wapman and Deborah Belle (a psychology professor), used this riddle to demonstrate the linger power of gender stereotype, as even self-defined feminists are inclined to neglect the possibility that the surgeon was a woman. The researchers conducted the experiment with two groups: 197 Boston University psychology students and 103 children from Brookline summer camps (ages 7 to 17). Only 15\% of the children and $14 \%$ of the students guessed that the surgeon was the mother. The adopted parent had the higher response (67 students), of which a surprising 51 assumed it was a gay parent. An alternative riddle, changing the words "father" with "mother" and "surgeon" with "nurse", produced no different results (Barlow, 2014; Wapman, 2014).

In our young lives, maybe we had that favorite toy that we carried everywhere we went. If you were a girl, it was probably a doll, if you were a boy, a car. (Blakemore and Centers, 2005) did a study that demonstrated that gender stereotype still exists regarding toys. Toys like dolls or domestic activities were normally associated to girls, while vehicles or action figures (transmitting aggression or violence) to boys. A psychologist at Harvard Medical School says, "Rigidly gendered toy marketing tells kids who they should be, how they should behave, and what they should be interested in" (Hains, 2015, pp. 3). This could be due to the evolution of the media industry during the 1980s, where limitations were removed, allowing media owners to profit by advertising children products by genre (Hains, 2015). The appearance of the personal computer was no different as it targeted mainly men and boys, helping to define the techie culture ("Geeks", "Nerds") and creating on the teachers' fake expectations that every student grows up playing computer and going to class with some knowledge. But then there was the case of Patricia Ordóñez: she did not own a computer but was convinced that she could study computer science or electrical engineering taking into consideration her strong math background. However, this proved to be insufficient and she eventually had to drop out technical field and pursue foreign language due to that assessment. Nevertheless, years later and with the help of a mentor, she completed her $\mathrm{PhD}$ in computer science and currently works as an assistant professor (Henn, 2014; Light, 1999).

The massive majority of engineers are believed to be men, so when we think of an engineer we normally associate that image to a man, thus turning women invisible. The campus environment itself can lead to singling out women by their genre and making them feel uncomfortable. We believe that gender associations removes women as possible candidates, as they are automatically excluded from 
hard and dangerous tasks (Faulkner, 2009, 2007, 2000; Jorgenson, 2002; McLoughlin, 2005; Tonso, $2006,1998)$. This lack of diversity also affects the $\mathrm{HCl}$ area. Designers and developers in practice use themselves as model users. The smart house, a product destined for everyone, is one of many designs that neglects something that the other gender needs/knows, as normally all of the household work is done by women (Berg, 1995).

A number of studies have investigated the time where that assumption was created. That is, it has been observed that from a young age, children have been associating some occupations to the male gender while others to the female gender. For example, a nurse or secretary are associated to a female occupation, whereas occupations such as police officer and firefighter are viewed as a male profession. Gender stereotype in children has been detected from a young age until they reach adolescence (i.e. from 2 to 16 years old). Nevertheless prior works did not detect the difference between the male and female children, encountering evidences that occupational stereotype reduces with age, but not to a point of eliminating it completely (Gettys and Cann, 1981; Miller and Budd, 1999; O’Keefe and Hyde, 1983).

\subsection{Implicit Gender Association}

"Implicit stereotypes are automatically activated and operate indirectly, and thus individuals may not be aware that they possess such beliefs. In contrast, explicit stereotypes are accessible to conscious awareness and are what individuals report when asked about group differences." (Dryden, 2013, p. 5) The implicit association (IAT) measures the associations between pairs of somatic or social categories thus detecting implicit stereotype (Greenwald et al., 1998; Nosek et al., 2002). Project Implicit, a non-profit organization, has released a variety of stereotypes and self-concepts (IAT), which explores the thoughts and feeling that occurs outside of conscious awareness or control ("Projectlmplicit," 2011).

(White and White, 2006) presented a study of occupation stereotype regarding college students by comparing the implicit (IAT) and explicit (Likert-type ratings scales) stereotypes of three occupations (engineer, accountant and elementary school teacher). Both implicit and explicit results detected the engineer as a male occupation while the elementary school teacher as a female occupation. The accountant occupation is what differs, while implicit was perceived to be a male, explicit was neutral (White and White, 2006). (Nosek et al., 2002) in turn, measured the implicit and explicit toward stereotype in social groups from a large sample ( \pm 600000 samples in 19 months), with nine IAT - implicit association test (e.g., race attitude faces, race attitude - names, age attitude - faces, age attitude - names, gender-science, gender - career, self-esteem, math - arts attitude, election 2000 attitude). The results contradict the (White and White, 2006) study regarding the relation between implicit and explicit attitudes. Both have been conceptualized as restricted and independent. (Nosek et al., 2002) data exhibit that implicit and explicit are more connected than formerly presumed (although this is often a weak connection). All in all, the relationship between implicit and explicit has been found small when correlating ages with names, moderated when correlating race with names and large with election 2000 candidates (Nosek et al., 2002).

If you have a brain, you have an inclination against one individual or group. A black gay woman took the implicit bias test (Race IAT), self-assured of what the result would be, but instead was astonished to discover an unconscious bias in benefit of white or light skin people. Later she discovered her unconscious bias was due to her past, as she was from a small English town, living next to white families where she received the most positive images. In her journey to discover more about the history of black people and enclose herself with positive images, that bias no longer was detected (CBC, 2017).

"The tests just test how quickly you are able to do as your told. [N]o one is saying that the test result that you would get from an implicit association would be an absolute, accurate indicator of your behaviour every time. What it can tell you is that this is your default position." (CBC, 2017, pp. 8)

In the present study, we considered implicit and explicit areas stereotypes in two types of departments (exact sciences versus arts and humanities - independent variable). The Exact Science area is practically dominated by male students while Arts and Humanities are the opposite. The present research seeks to examine how social attitudes interfere with the number of women in technical fields. More specifically, this work, aims to understand if it exists an occupational stereotype among students of first year that perhaps could interfere in their choice of area.

\section{METHOD}

\subsection{Participants}

Forty one students (22 males and 19 females, ages from 17 to 26) enrolled in the departments of Exact Science and Arts and Humanities at the local University participated in the Study. Nine participants had previously been excluded from further analysis, as they did not belong to the first year of college. We did so because during their first year, the probability to change to a different course is higher than in the following years. All participants received a small gastronomic gratification for their participation. 


\subsection{Materials and Apparatus}

The study was conducted as part of a class. The students were informed that the purpose of the study was to understand the association of gender to specific occupations. Participants were asked to sit in front of a computer.

The materials and procedures of this study were approximately parallel to Project Implicit ("Projectlmplicit," 2011). Specifically, we transformed the 8 words of each category to 5 words, changed its category title to two of the current University departments and its courses: five male words (father, uncle, man, grandfather and son), five female words (mother, aunt, women, grandmother and daughter), five exact science words (biology, biochemistry, economic, engineering and mathematics) and five arts and humanities words (culture, education, communication, languages and psychology).

\subsection{Design and Hypotheses}

All participants responded to two pairs of target departments and gender attributes, randomly assigned and were arbitrarily assigned to one of the conditions (AB or BA, where A stands for the control condition and $B$ for the experimental), with a gap between conditions to break the familiarity of the first condition. In this study, exact science + male \& arts and humanities + female, followed by exact science + female $\&$ arts and humanities + male. A difference was expected between exact science + male and exact science + female. A research hypothesis was created to determine if students took less time associating exact sciences areas to male than to female and arts and humanities areas to female than to male.

\subsection{Procedure}

Participants were asked to first read and sign a written consent form. Each participant was seated at a table with a computer in front of them. All instructions were presented both verbally and visually.

The Implicit Association Test: Similar to project implicit, the IAT consisted of a total of nine blocks: (1) Arts and Humanities/Exact Science discrimination (20 trials); (2) Male/Female discrimination (20 trials); (3) combination, common gender stereotype: Exact Science and Male were categorized with the same response key (20 trials); (4) repeated combined block (20 trials); (5) break; (6) Arts and Humanities/Exact Science discrimination (20 trials); (7) Reverse Male/Female discrimination (20 trials); (8) combination, uncommon gender stereotype: Exact Science and Female were categorized with the same response key (20 trials); (9) repeated combined block (20 trials); The block with individuals categories $(1,2,6$ and 7) were considered practice trials and were not included in the analyses. Each block was anticipated by a set of instructions regarding the categorization task and the suitable key reaction. Participants were asked to respond as quickly and accurately as possible, by pressing one of two keys on a keyboard. If the target word belonged to the category on the left side of the screen, the letter key "E" had to be pressed. If the target world belonged to the category on the right side of the screen, the letter key "I" had to be pressed. Each target word would wait $500 \mathrm{~ms}$ before appearing in the center of the screen, where it would continue until the participant responded.

Explicit attitude measures: Following the IAT, the participants completed a questionnaire focus on their beliefs, attitudes and opinions (questions from Project Implicit). Participants were asked to rate on a 7 and 5-point Likert scale, how they associate the Departments (Exact Science and Arts and Humanities) regarding their numbers of male and female, how they evaluate their attitude regarding those departments and their importance regarding high position, interests, work time, incentive, preference and to be specialist in a department. The final 5 questions were about personal information (age, genre, course, the optional courses to which students applied at their college application and which was their highest grade/subject in high school), to try to understand the reason for choosing that area. Finally, the participants were debriefed about the experiment and thanked for their participation. Both parts, the questionnaire and IAT, took 15-20 min to complete.

\section{RESULTS}

We analyzed every key interaction in both conditions to determine in which study they took longer to associate. Half of the participants started with exact science + female associations and the remaining half with exact science + male. The time range of the study varied from 4 minutes and 36 seconds to 8 minutes and 25 seconds (the time to sign the consent form and filling the questionnaire was excluded). Results showed that the participants took less time associating exact science with male than exact science with female $(p<.05)$. Students registered in a median of 24385.50 milliseconds (approx. 24 seconds) for the study associating exact science with male and 28948 milliseconds (approx. 29 seconds) in the other condition, associating exact science with female.

The data indicates that female students of arts and humanities associated exact science with female quicker. In other words, 13 students had a faster time associating female with exact science than male to exact science. Analyzing further regarding the area and gender, with 10 out of 27 from exact science and 3 out of 14 from arts and humanities, as 
for gender, 7 out of 19 were female and 6 out of 22 were male.

A deeper examination regarding the department and gender revealed, that in this study the difference of time between male and female students did not have any significant effect. As the difference between the courses, the questionnaire showed what was foreseen. The overall results determined that in the department of Arts and Humanities, female were the majority of students, however in the department of exact science neither male nor female were the majority. The gender and area were analyzed, males and exact science students consider the arts and humanities has a majority of female students while exact science has a majority of male. The female students considered neither male nor female dominated both departments. The same occurred for the students in the department of arts and humanities as they considered both areas neither male nor female ruled.

\section{DISCUSSION}

The difference between the implicit and explicit results presents an interesting example of how our conscious and unconscious differs and how our future life opportunities are probably affected by our educational choices. The studies mentioned above, lead us to assume that the stereotype regarding women in technical fields would disappear with age (Gettys and Cann, 1981; Miller and Budd, 1999; O'Keefe and Hyde, 1983). Our results suggest that this is not the case, however we cannot confirm as we will need to follow participants through their growth.

The study of (White and White, 2006) and (Nosek et al., 2002) compared with this one, is similar as both detect in an implicit and explicit way. Both studies agree regarding the implicit in a technical area is associated to male and a non-technical area to female. The explicit way is where they disagree: in the (White and White, 2006) study, the explicit was the same as the implicit attitude, the technical area was associated to male and non-technical area to female; in the (Nosek et al., 2002) study also revealed the same, with one small detail: after the age of 50 , the implicit association of technical areas with male increases while explicit do not, it distances; in this study, the non-technical area was associated to female but the technical area was neither female nor male. An occupation which is mentally intended to one gender only, can eventually stop them to look outside their comfort zone, or what society dictates as their comfort zone. If there is a conflict between actions (what we've done) and attitudes (what we really believe), that causes a cognitive dissonance. The fact that female students believe that they can choose to study in any area, does not imply that they will follow an area that is generally assigned to the male gender.

\subsection{Limitations}

Some limitations of the study were found. Due to the size of the sample, the results describe show a small effect. The examiner made every effort to reinforce the break between conditions, however some participants ignored the break completely. Moreover, it is possible that some students did not see the word and kept interacting with the keywords to finish as quickly as possible.

In summary, the present study seeks to examine how social attitudes interfere with the number of women in technical fields. The hypothesis is that the students associate technical fields with male rather than to female, confirming it but in a small sample, having no differences in relation to the student's gender or department. This leads to the fact that our assumptions might contribute to our future occupation/career, as the cognitive dissonance theory states the idea that we seek firmness in our beliefs and attitudes where two cognitions are incompatible.

\section{CONCLUSION \& FUTURE WORK}

"... when it comes to gender, we fixate on women's reproductive functioning, and we sort of allot competence to men." (Barlow, 2014, pp. 8)

The findings of this study confirm there is an occupational stereotype among students of first year that perhaps was the cause of their choice of area. They unconsciously associate technical fields to male then to female, however consciously some associate a balance of genders between the areas.

The gender inequality in technology is a major problem in modern society as it constrains our innovation capacities and our ability to grow. Women are normally excluded from design and are prevented to use technologies because they are indirectly centered on a model of a male or masculine user. We believe that diversity in the working classes can lead to creativity, productivity and innovation. One lesson we might take from this study is that if we come to an early awareness of this assumptions the number of women in technical fields could increase, creating a balance in the gender occupation.

Our next step would be to create an analytic dashboard that would exhibit students of the gender inequality, specifically the number of female students that applied to technical areas over the past years and colleges. Our goal is to create an uncomfortable tension. In order to restore balance, a necessary action is required for example: 1 . they can change their behavior as to pursue technical fields; 2. or justify their pursuit of a non-technical 
fields by changing theirs beliefs that only male should pursuit technical fields; 3 . or adding knowledge that if they pursue it they might not be successful.

\section{ACKNOWLEDGMENT}

This research was supported by ARDITI (Agência Regional para o Desenvolvimento da Investigação, Tecnologia e Inovação), Doctoral Grant under the Project M14-20 - 09-5369-FSE-000001.

\section{REFERENCES}

Berg, A.-J., 1995. A Gendered Socio-Technical Construction: The Smart House | Keele University [WWW Document]. URL http://lists.lib.keele.ac.uk/items/953E16B64924-FE6B-81C5-647E37231CB6.html (accessed 6.4.18).

Barlow, R., 2014. BU Research: A Riddle Reveals Depth of Gender Bias [WWW Document]. BU Today. URL http://www.bu.edu/today/2014/bu-researchriddle-reveals-the-depth-of-gender-bias/ (accessed 1.26.17).

Beddoes, K.D., 2012. Feminist Scholarship in Engineering Education: Challenges and Tensions. Eng. Stud. 4, 205-232. https://doi.org/10.1080/19378629.2012.693 932

Bix, A.S., 2004. From

\&amp;quot;Engineeresses\&amp;quot; to \&amp;quot;Girl Engineers\&amp;quot; to \&amp;quot;Good Engineers\&amp;quot;: A History of Women's U.S. Engineering Education. NWSA J. 16, 27-49. https://doi.org/10.1353/nwsa.2004.0028

Blakemore, J.E.O., Centers, R.E., 2005. Characteristics of Boys' and Girls' Toys. Sex Roles 53, 619-633. https://doi.org/10.1007/s11199-005-7729-0

Blickenstaff, clark J., 2005. Women and science careers: leaky pipeline or gender filter? Gend. Educ. 17, 369-386. https://doi.org/10.1080/0954025050014507 2

Brainard, S.G., Carlin, L., 1998. A six-year longitudinal study of undergraduate women in engineering and science. J. Eng. Educ. 87, 369-375.

Dryden, K.L., 2013. Implicit and Explicit Gender Stereotypes and Their Relationship to SelfConcept. University of North Carolina at Chapel Hill.

Faulkner, W., 2009. Doing gender in engineering workplace cultures. II. Gender in/authenticity and the in/visibility paradox. Eng. Stud. 1, 169-189. https://doi.org/10.1080/1937862090322505 9

Faulkner, W., 2007. 'Nuts and Bolts and People': Gender-Troubled Engineering Identities. Soc. Stud. Sci. 37, 331-356. https://doi.org/10.1177/0306312706072175

Faulkner, W., 2000. Dualisms, Hierarchies and Gender in Engineering. Soc. Stud. Sci. 30, 759-792.

https://doi.org/10.1177/0306312000300050 05

Gettys, L.D., Cann, A., 1981. Children's perceptions of occupational sex stereotypes. Sex Roles 7, 301-308.

Greenwald, A.G., McGhee, D.E., Schwartz, J.L., 1998. Measuring individual differences in implicit cognition: the implicit association test. J. Pers. Soc. Psychol. 74, 1464.

Hains, R., 2015. The problem with separate toys for girls and boys - The Boston Globe [WWW Document]. BostonGlobe.com. URL https://www.bostonglobe.com/magazine/20 $15 / 02 / 27 /$ the-problem-with-separate-toysfor-girls-andboys/2ul7Qp0d3oYrTNj3cGkiEM/story.html (accessed 1.26.17).

Henn, S., 2014. When Women Stopped Coding [WWW Document]. NPR.org. URL http://www.npr.org/sections/money/2014/10 /21/357629765/when-women-stoppedcoding (accessed 1.17.17).

Jorgenson, J., 2002. Engineering selves negotiating gender and identity in technical work. Manag. Commun. Q. 15, 350-380.

Light, J.S., 1999. When Computers Were Women. ResearchGate 40, 455-483. https://doi.org/10.1353/tech.1999.0128

Literary Devices, 2015. Riddle - Examples and Definition of Riddle [WWW Document]. Lit. Devices. URL http://literarydevices.net/riddle/ (accessed 1.26.17).

CBC, 2017. How to overcome your unconscious biases | CBC Radio [WWW Document]. CBC. URL http://www.cbc.ca/radio/tapestry/facing-ourshadows-1.4037913/how-to-overcomeyour-unconscious-biases- 1.4038641 (accessed 3.9.18).

McLoughlin, L.A., 2005. Spotlighting: Emergent gender bias in undergraduate engineering education. J. Eng. Educ. 94, 373.

Miller, L., Budd, J., 1999. The Development of Occupational Sex-role Stereotypes, Occupational Preferences and Academic Subject Preferences in Children at Ages 8, 12 and 16. Educ. Psychol. 19, 17-35. https://doi.org/10.1080/0144341990190102

Nosek, B.A., Banaji, M., Greenwald, A.G., 2002. Harvesting implicit group attitudes and beliefs from a demonstration web site. 
Group Dyn. Theory Res. Pract. 6, 101115. https://doi.org/10.1037//10892699.6.1.101

O'Keefe, E.S., Hyde, J.S., 1983. The development of occupational sex-role stereotypes: The effects of gender stability and age. Sex Roles 9, 481-492.

ProjectImplicit [WWW Document], 2011. URL https://implicit.harvard.edu/implicit/ (accessed 1.26.17).

Tonso, K.L., 2006. Student Engineers and Engineer Identity: Campus Engineer Identities as Figured World. Cult. Stud. Sci. Educ. 1, 273-307. https://doi.org/10.1007/s11422-005-9009-2

Tonso, K.L., 1998. Engineering Gender-Gendering Engineering: What About Women in NerdDom?.

Wapman, M., 2014. Riddle me this. Mothers Nat.

White, M.J., White, G.B., 2006. Implicit and Explicit Occupational Gender Stereotypes. Sex Roles 55, 259-266.

https://doi.org/10.1007/s11199-006-9078-z 